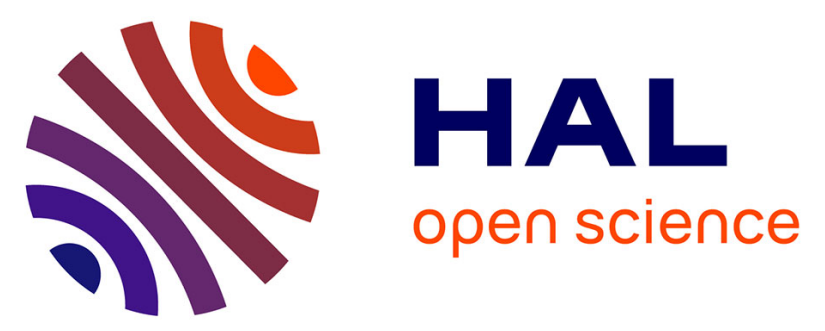

\title{
Influence of annealing time on structural and magnetic properties of rapid thermally annealed FePt films
}

\author{
C Brombacher, C Schubert, K Neupert, M Kehr, J Donges, M Albrecht
}

\section{To cite this version:}

C Brombacher, C Schubert, K Neupert, M Kehr, J Donges, et al.. Influence of annealing time on structural and magnetic properties of rapid thermally annealed FePt films. Journal of Physics D: Applied Physics, 2011, 44 (35), pp.355001. 10.1088/0022-3727/44/35/355001 . hal-00649054

\section{HAL Id: hal-00649054 https://hal.science/hal-00649054}

Submitted on 7 Dec 2011

HAL is a multi-disciplinary open access archive for the deposit and dissemination of scientific research documents, whether they are published or not. The documents may come from teaching and research institutions in France or abroad, or from public or private research centers.
L'archive ouverte pluridisciplinaire HAL, est destinée au dépôt et à la diffusion de documents scientifiques de niveau recherche, publiés ou non, émanant des établissements d'enseignement et de recherche français ou étrangers, des laboratoires publics ou privés. 


\title{
Influence of annealing time on structural and magnetic properties of rapid thermally annealed FePt films
}

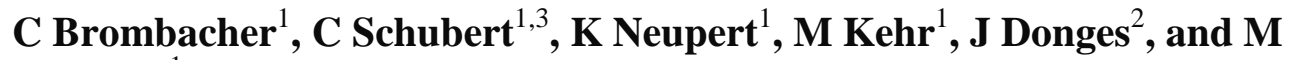 \\ Albrecht ${ }^{1}$ \\ ${ }^{1}$ Institute of Physics, Chemnitz University of Technology, D-09107 Chemnitz, Germany \\ ${ }^{2}$ HASYLAB, DESY, D-22603 Hamburg, Germany \\ ${ }^{3}$ Corresponding author: christian.schubert@ physik.tu-chemnitz.de
}

\begin{abstract}
L} 1_{0}$ ordered FePt films with (001) texture and out-of-plane magnetic easy axis were fabricated by rapid thermal annealing of chemically disordered FePt thin films grown on amorphous $\mathrm{SiO}_{2}$ - substrates. While keeping the annealing temperature fixed at $800^{\circ} \mathrm{C}$, the influence of the annealing time was investigated in the regime between $5 \mathrm{~s}$ and $300 \mathrm{~s}$. The evolution of the $a$ and $c$ lattice parameter indicate that in-plane strain is a key factor for the formation of the (001) texture. A large tetragonal distortion is found after an annealing time of $5 \mathrm{~s}$ whereas a relaxation of the lattice parameters towards bulk values is found for longer annealing times. The dependence of the magnetic properties on the annealing time will be discussed with respect to the observed variation of the structure and morphology of the annealed FePt films. 75.30.Cr, 75.30.Gw, 75.50.Bb, 75.50.Ss, 75.50.Vv, 75.70.Ak, 61.05.cp, 61.66.Dk
\end{abstract}

\section{Introduction}

$\mathrm{L}_{0}$ ordered FePt films exhibit large uniaxial magnetocrystalline anisotropy of $\sim 10^{8} \mathrm{erg} / \mathrm{cm}^{3}$ [1] and are thus considered promising candidates for future application in magnetic storage devices [2 - 4], magnetic sensors [5] or as permanent magnets [6]. For most applications, a controlled crystallographic orientation of the chemically ordered FePt films in particular on amorphous substrates is a key requirement. In this regard, (001) textured FePt films revealing perpendicular magnetic anisotropy have been in the focus of current research. Various approaches to obtain (001) texture like the usage of appropriate substrates or seed-layers [7 - 11], ion irradiation [12] or annealing of Pt/Fe bilayers under hydrogen atmosphere [13] do exist. However, especially rapid thermal annealing (RTA) of Fe/Pt multilayers has attracted much attention since pronounced perpendicular anisotropy and coercivities up to 10 kOe [14] as well as a high degree of chemical order [15] and high energy products exceeding 40 MGOe [16] can be achieved on amorphous glass substrates within very short annealing times. The mechanisms involved in the (001) texture formation were discussed by Kim et al. and it is suggested that tensile in-plane strains during the order/disorder transformation favor the growth of (001) textured grains $[17,18]$.

In this study, $\mathrm{FePt}$ films deposited via magnetron-sputtering at room-temperature were processed by RTA. The influence of the annealing time on the structural and magnetic properties as well as possible mechanisms for the evolution of the (001) texture will be discussed.

\section{Experimental}

5-nm-thick $\mathrm{Fe}_{52} \mathrm{Pt}_{48}$ films were sputter-deposited onto thermally oxidized $\mathrm{Si}(100)$ substrates with a $\mathrm{SiO}_{2}$ thickness of $100 \mathrm{~nm}$ using an Fe target covered with $\mathrm{Pt}$ chips. The $\mathrm{Ar}^{+}$sputter pressure was adjusted to $3.5 \times 10^{-3}$ mbar for all depositions and the composition of the alloy was later on verified by Rutherford Backscattering (RBS). The samples were then transferred to a separate chamber, in which the RTA process was applied under $\mathrm{N}_{2}$ atmosphere. The heating rate was fixed to $400^{\circ} \mathrm{C} / \mathrm{s}$, the annealing temperature to $800^{\circ} \mathrm{C}$ and the annealing time, $t_{\text {anneal }}$, was varied between $5 \mathrm{~s}$ and $300 \mathrm{~s}$. The structural properties of the samples were investigated by x-ray diffraction using beamline G3 at HASYLAB, 
DESY. Integral magnetic characterization was performed using superconducting quantum interference device (SQUID) magnetometry, while the topography and local domain configuration was analyzed by combined atomic and magnetic force microscopy (AFM/MFM) at room temperature.

\section{Results and Discussion}

X-ray diffraction patterns obtained in $\theta-2 \theta$ geometry show both fundamental and superstructure peaks related to (001) textured FePt films for all annealing times (figure 1). Even after a short annealing time of $5 \mathrm{~s}$, the (001) superstructure and (002) fundamental peaks are dominant compared to the (111) reflection and indicate a pronounced (001) texture. Please note that in these diffraction experiments the illuminated sample area was the same for all samples. After an annealing time of $30 \mathrm{~s}$, the integral intensity of the peaks reaches its maximum corresponding to the best (001) texture quality achieved for the investigated sample series. For annealing times larger than $150 \mathrm{~s}$, additional diffraction peaks appear which can most likely be attributed to silicide, oxide or even silicate formation at the $\mathrm{FePt} / \mathrm{SiO}_{2}$ interface during the RTA process. The full width at half maximum (FWHM) of the (001) peak was used to extract the perpendicular coherence length, $L_{\text {perp. }}$. A value of $L_{\text {perp }}$ up to $30 \mathrm{~nm}$ which is about 6 times larger than the initial film thickness was found for the longest annealing times and indicates a change in the film morphology from continuous to island-like (see discussion on AFM images below). The intensity ratio $I_{001} / I_{002}$ of the superstructure and fundamental peak was used to extract the long range chemical order parameter $\mathrm{S}$ according to following expression

$$
S^{2}=\frac{I_{001}}{I_{002}} \cdot\left(\frac{F_{002}}{F_{001}}\right)^{2} \cdot \frac{(L \cdot A \cdot D)_{002}}{(L \cdot A \cdot D)_{001}}
$$

where $F$ is the structure factor, $L$ the Lorentz factor, $A$ the absorption factor and $D$ the temperature factor. The absorption factor $A$ was approximated to be proportional to $1 / \sin \theta$ and to accurately determine $D$ and $L$, all reflections up to the $4^{\text {th }}$ order, including their corresponding rocking curves have been measured for samples with $30 \mathrm{~s} \leq \mathrm{t}_{\text {anneal }} \leq 150 \mathrm{~s}$. Since the mosaicity of these samples was found to be around $3^{\circ}$ and the beam divergence was $0.9 \times 0.4 \mathrm{mrad}$, the Lorentz factor $L$ can be approximated using the expression $L=\frac{1}{\sin 2 \theta \sin \theta} . D$ was determined according to the routine proposed by Berg and Cohen [19], but since values close to 1 have been obtained for all (001) and (002) peaks, the influence of $D$ was neglected. With these values a constant ordering parameter of $S=0.88 \pm 0.1$ could be determined. No variation of $S$ with annealing time was observed, indicating the strong correlation between the (001) texture formation and the first order phase transformation.

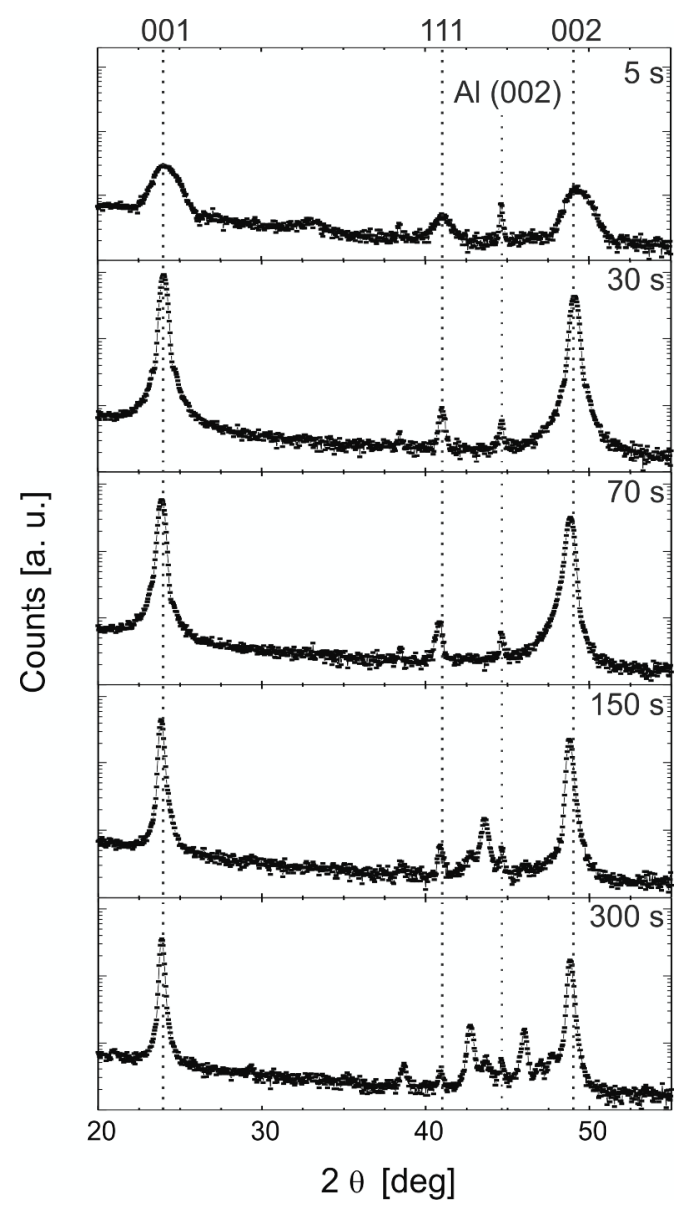

Figure 1: X-ray $\theta-2 \theta$ diffraction patterns of 5-nmthick FePt films after RTA to $800^{\circ} \mathrm{C}$ for annealing times varying between $5 \mathrm{~s}$ and $300 \mathrm{~s}$ plotted in a logarithmic scale. The positions of the 001,111 and 002 reflections of bulk FePt, as well as of the 002 reflection of the Al sample holder are indicated. 
To get a deeper insight into the origin of the (001) texture formation, the $c$ lattice parameter was extracted from the $(001)$ diffraction peaks $(1 \leq 1 \leq 4)$ and the $a$ lattice parameter was extracted via tilting the sample to $\chi_{1}=53.66^{\circ}$ and $\chi_{2}=43.87^{\circ}$ allowing to measure in asymmetric $\theta-2 \theta$ geometry the peaks corresponding to the (111) and (202) planes of the (001) textured grains, respectively. This method allows the determination the tetragonal distortion induced by the RTA process. After an annealing time of $5 \mathrm{~s}$, the $c$-axis is substantially contracted while the $a$-axis is expanded compared to the bulk values of $\mathrm{L}_{0}$ FePt (figure $2(\mathrm{a}, \mathrm{b})$ ). Longer annealing times lead to a relaxation of the lattice parameters towards its bulk values and after an annealing time of $150 \mathrm{~s}$ no further change of the lattice parameters is observed. Concerning relevant mechanisms for the formation of the (001) texture, the strong tetragonal distortion is an indication of in-plane strain leading to the energetically favorable growth of (001) oriented grains, with the longer axis of the tetragonal distorted $f c t$ lattice pointing in direction of the strain. A likely origin of this in-plane strain is the effective volume shrinkage occurring during the rapid recrystallization process with the annihilation of defects and grain boundaries [20].

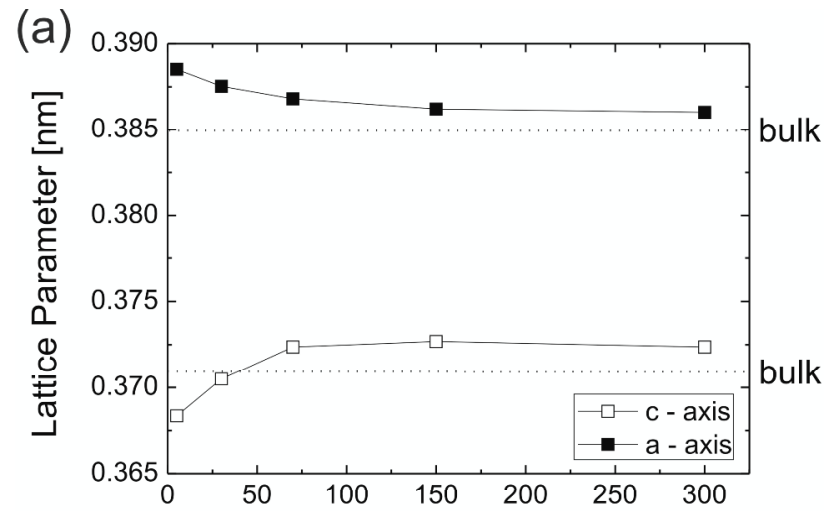

Figure 2: Dependence of (a) the $a$ and $c$ lattice parameter and (b) the $c / a$ ratio on the annealing time. The values of bulk $\mathrm{L1}_{0}$ FePt are indicated.

(b)

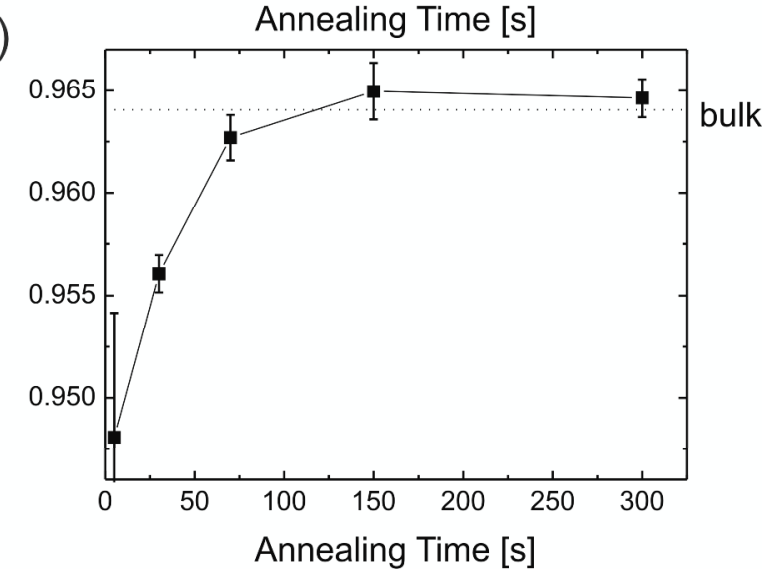

Figure 3 is showing the evolution of the topography and the corresponding magnetic domain configuration after RTA for different annealing times. The annealing process is modifying the morphology of the FePt films from smooth continuous films in the as-grown state ( $\mathrm{rms}$ roughness $=0.6 \mathrm{~nm}$ ) towards an island-like morphology. The onset of this dewetting behavior starts around an annealing time of $5 \mathrm{~s}$ and cracks and voids are observable (figure 3 (a)). For an annealing time of $30 \mathrm{~s}$ (figure 3(b)), the island-like morphology is fully developed (rms roughness $=3.0 \mathrm{~nm}$ ) and islands with decreasing diameter and increasing height are observed when the annealing time is further increased (rms roughness $=12.3$ $\mathrm{nm}$ after $t_{\text {anneal }}=150 \mathrm{~s}$ ). Since the annealing temperature is $800^{\circ} \mathrm{C}$ during the RTA process and thus exceeding the Curie-temperature typically observed in thin FePt films [21], the FePt films are in a demagnetized state after cooling down to room temperature. Annealing times of around $t_{\text {anneal }}=30 \mathrm{~s}$ are required until all $\mathrm{FePt}$ islands reveal pronounced dark and bright contrast indicating perpendicular magnetic anisotropy (figure 3 (b)). Islands with a diameter below $100 \mathrm{~nm}$ primarily show a singledomain state and multi-domain states are formed within larger islands. Although the coercivity of particulate $\mathrm{L}_{0}$ ordered FePt films is typically well above the stray field of the magnetic tip, several tipinduced switching events in multi-domain islands can be identified (figure 3 (b)). This finding indicates a high inter-island exchange coupling with low pinning fields allowing the depinning of existing domain walls by the stray field of the magnetic tip. For long annealing times $\left(t_{\text {anneal }} \geq 150 \mathrm{~s}\right)$ only a reduced number of islands are revealing magnetic contrast (figure 3 (c)) which is in agreement with the proposed silicide formation leading to a degradation of the magnetic properties. 

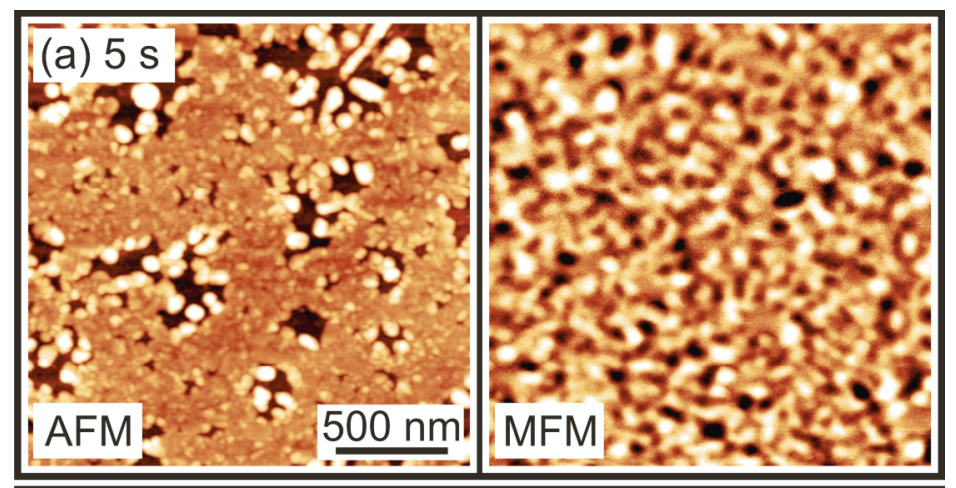

Figure 3: AFM and corresponding MFM images of 5-nm-thick FePt films after RTA for (a) $5 \mathrm{~s}$, (b) $30 \mathrm{~s}$, and (c) $150 \mathrm{~s}$. Some FePt grains are highlighted for a better comparison between the topography and the magnetic domain configuration.
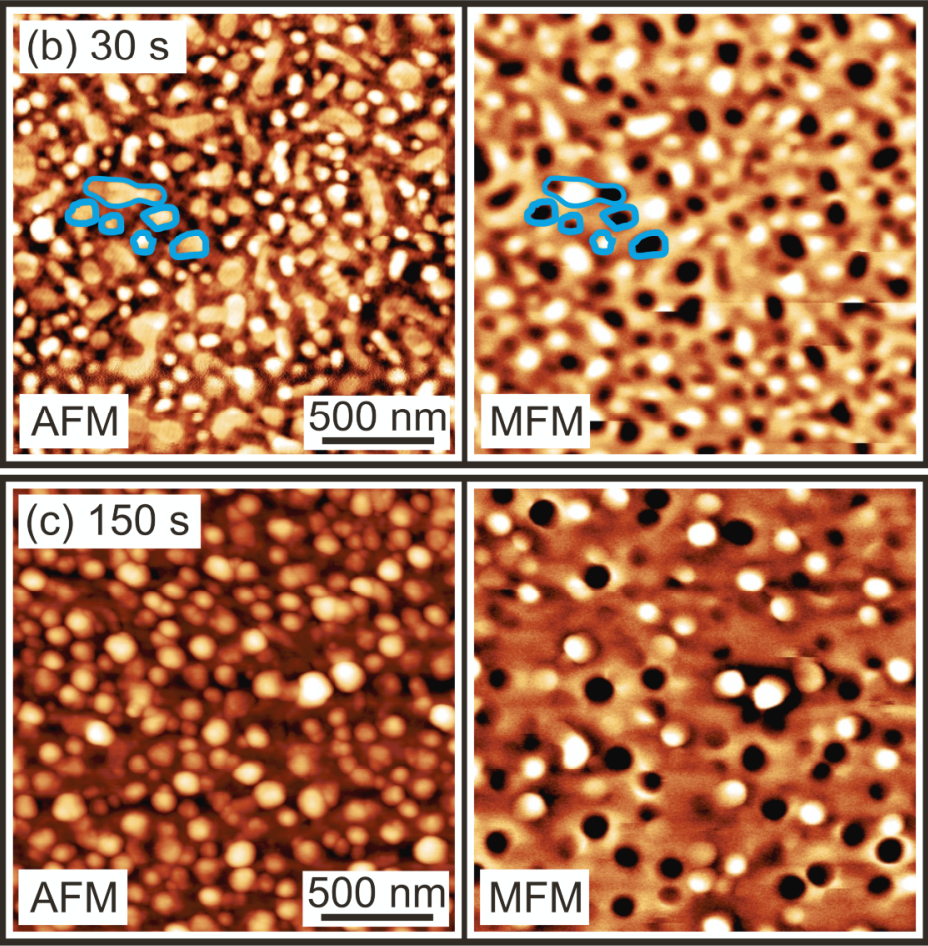

A series of magnetic hysteresis loop was measured by SQUID magnetometry in out-of-plane and in plane geometry as presented in figure 4 . While an in-plane easy axis with $K_{\text {eff }}=-(3.6 \pm 0.4) \times 10^{6}$ ergs $/ \mathrm{cm}^{3}$ is observed for the as-grown chemically disordered (A1 phase) FePt film which is given by the shape anisotropy $K_{\mathrm{S}}=2 \pi M_{\mathrm{S}}^{2}=(3.4 \pm 0.4) \times 10^{6} \mathrm{ergs} / \mathrm{cm}^{3}$, an annealing time of $5 \mathrm{~s}$ is already sufficient to turn the easy axis of the magnetization into the out-of-plane direction. Linear extrapolation of the hard axis loop allowed to estimate the anisotropy field $H_{\mathrm{a}}$ for all samples which was used to determine an approximate effective magnetic anisotropy constant $K_{\text {eff }}=1 / 2 M_{\mathrm{s}} H_{\mathrm{a}}$, which was showing a maximum of around $(70 \pm 40) \times 10^{6} \mathrm{ergs} / \mathrm{cm}^{3}$ after an annealing time of $30 \mathrm{~s}$. This maximum coincides with the maximum of the integrated intensity of the diffraction peaks. The coercivity, however, is not exhibiting a clear maximum, but increases with annealing time and saturates at around $45 \mathrm{kOe}$ as summarized in figure 5 (a). As the saturation field along the easy axis is close to $70 \mathrm{kOe}$, additional magnetic measurements (not shown) were carried out on a VSM magnetometer allowing higher applied magnetic fields up to $90 \mathrm{kOe}$. These measurements confirmed that fields of $70 \mathrm{kOe}$ are sufficient to fully saturate the samples. The high coercivity and broad switching field distribution is in good agreement with the observed particulate morphology, since nucleating domains in particulate FePt films is typically leading to substantially higher coercivities and switching field distributions as compared to continuous films, in which the reversal is dominated by nucleation followed by domain wall propagation [22]. In this regard figure 4 (c) reveals two step-like reversals, which can be correlated with the underlying microstructure of the sample (see figure 3 (b)), where larger continuous areas are separated by independent islands.

The $\mathrm{A} 1-\mathrm{L}_{0}$ phase transformation is accompanied by a pronounced increase in saturation magnetization (figure $5(\mathrm{~b})$ ) and after $t_{\text {anneal }}=5 \mathrm{~s}$, a value of $\mathrm{M}_{\mathrm{S}}=(1060 \pm 130) \mathrm{emu} / \mathrm{cm}^{3}$ was determined which is consistent with the values reported in literature [23]. This increase in saturation magnetization is not a result of the ordering transformation itself as the $\mathrm{M}_{\mathrm{s}}$ value for the disordered FePt phase is rather similar, but due to annihiliation of defects and removal of impurities during the RTA process. The 
following decrease in $M_{\mathrm{S}}$ for $t_{\text {anneal }} \geq 150 \mathrm{~s}$ is attributed to a possible intermixing at the $\mathrm{FePt} / \mathrm{SiO}_{2}$ interface as already discussed.

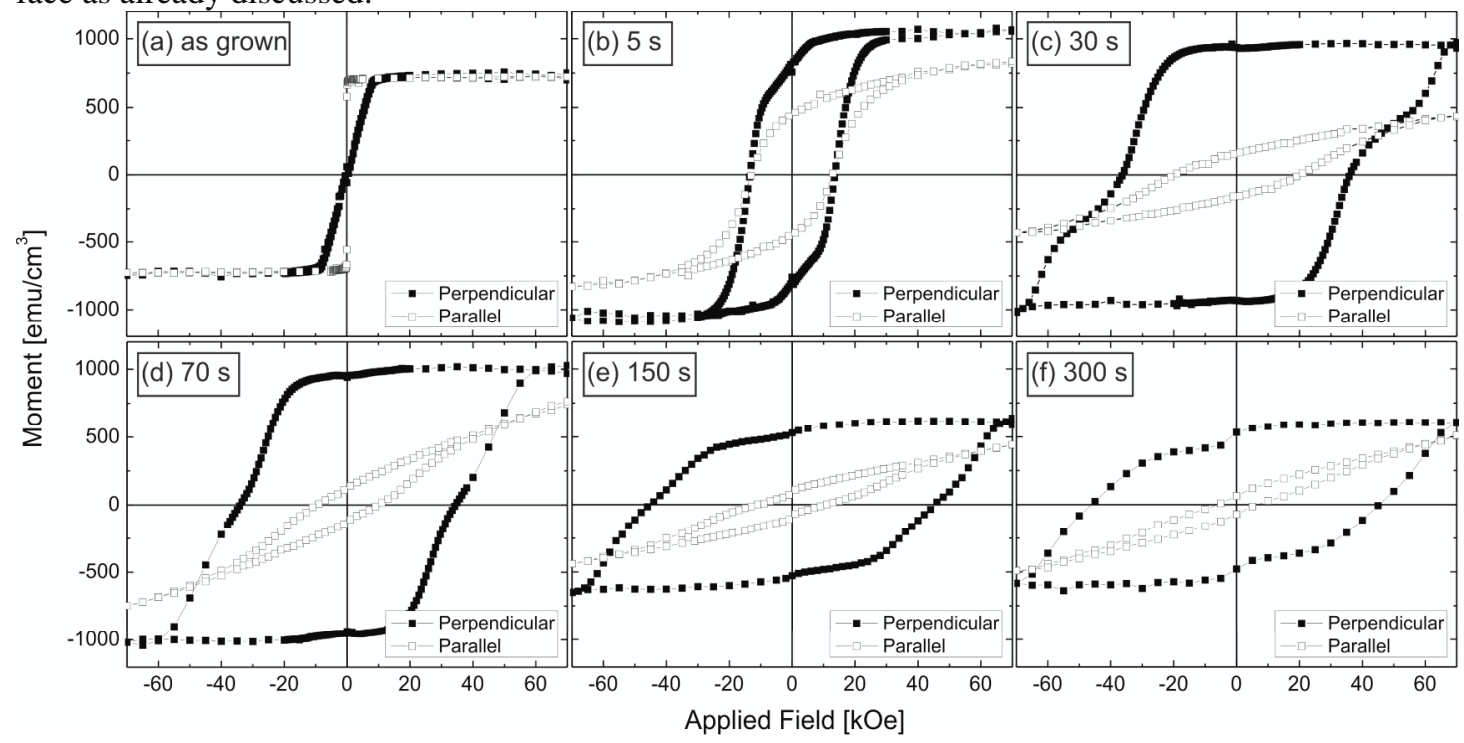

Figure 4: Hysteresis loops obtained by SQUID magnetometry of $5 \mathrm{~nm}$ FePt in (a) the as-grown state and after RTA for (b) $5 \mathrm{~s}$, (c) $30 \mathrm{~s}$, (d) $70 \mathrm{~s}$, (e) $150 \mathrm{~s}$ and (f) $300 \mathrm{~s}$ with the magnetic field applied perpendicular and parallel to the sample plane.

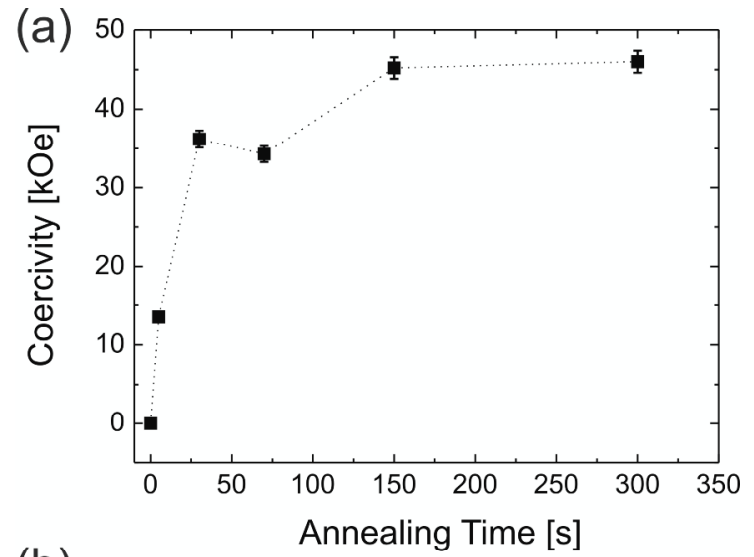

Figure 5: Dependence of (a) the coercivity and (b) the saturation magnetization on the annealing time.

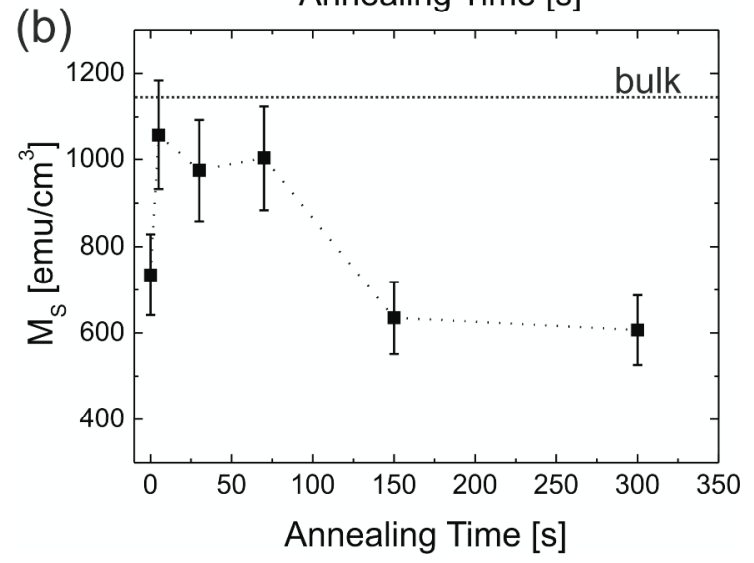

\section{Conclusion}

Rapid thermal annealing was used to transform chemically disordered FePt films into $\mathrm{L}_{0}$ ordered and (001) textured FePt films on amorphous $\mathrm{SiO}_{2}$ - substrates by applying an annealing temperature of $800^{\circ} \mathrm{C}$. The evolution of the lattice parameters as a function of annealing time was studied indicating that in-plane strain is present during the first $100 \mathrm{~s}$ of the annealing process favoring the growth of (001) oriented grains. This in-plane strain might be driven by the effective volume shrinkage occurring during the rapid recrystallization process with the annihilation of defects and grain boundaries.

Furthermore, a strong effective perpendicular magnetic anisotropy of up to $70 \mathrm{x} 10^{6} \mathrm{ergs} / \mathrm{cm}^{3}$ with a coercivity of about $35 \mathrm{kOe}$ was obtained for an annealing time of $30 \mathrm{~s}$. Under these RTA conditions a pronounced dewetting behavior of the film is observed leading to particulate FePt films. 


\section{Acknowledgments}

We kindly thank Volker Neu (IFW Dresden) for valuable scientific comments and the opportunity to perform VSM measurements. Part of this research was carried out at HASYLAB (beamline G3) at DESY (Hamburg, Germany) and the financial support by HASYLAB (project No. I-20090119) is gratefully acknowledged.

\section{References}

[1] Farrow R F C, Weller D, Marks R F, Toney M F, Cebollada A and Harp G R 1996 J. Appl. Phys. 795967.

[2] Weller D and Doerner M F 2000 Annu. Rev. Mater. Sci. 30611.

[3] Piramanayagam S N 2007 J. Appl. Phys. 102011301.

[4] Kim H, Noh J S, Roh J W, Chun D W, Kim S, Jung S H, Kang H K, Jeong W Y and Lee W 2011 Nanoscale Res. Lett. 613.

[5] Ohmori K, Tan K, Itoi K, Nagasu K, Uemichi Y, Aizawa T and Yamauchi R 2008 IEEE Trans. Magn. 443977.

[6] Liu J P, Luo C P, Liu Y and Sellmyer D J 1998 Appl. Phys. Lett. 72483.

[7] Weisheit M, Schultz L and Fähler S 2007 Thin Solid Films 5153952.

[8] Sun A C, Kuo P C, Hsu J H, Huang H L and Sun J M 2005 J. Appl. Phys. 98076109.

[9] Y. Xu, J. S. Chen, and J. P. Wang, Appl. Phys. Lett. 80, 3325 (2002).

[10] Peng Y, Zhu J G and Laughlin D E 2006 J. Appl. Phys. 9908 F907.

[11] Makarov D, Brombacher C, Liscio F, Maret M, Parlinska M, Meier S, Kappenberger P and Albrecht M 2008 J. Appl. Phys. 103053903.

[12] Bernas H, Attané J Ph, Heinig K H, Halley D, Ravelosona D, Marty A, Auric P, Chappert C and Samson Y 2003 Phys. Rev. Lett. 91077203.

[13] Ikemoto J I, Imai Y and Nakagawa S 2008 IEEE Trans. Magn. 443543.

[14] Yan M L, Powers N and Sellmyer D J 2003 J. Appl. Phys. 938292.

[15] Yamane H, Narisawa T, Hasegawa T and Ishio S 2010 J. Appl. Phys. 108113923.

[16] Liu J P, Luo C P, Liu Y and Sellmyer D J 1998 Appl. Phys. Lett. 72483.

[17] Kim J S, Koo Y M, Lee B J and Lee S R 2006 J. Appl. Phys. 99053906.

[18] Kim J S, Koo Y M and Shin N 2006 J. Appl. Phys. 100093909.

[19] Berg H and Cohen B J 1972 Metall. Trans. 31797.

[20] Zeiger W, Brückner W, Schumann J, Pitschke W and Worch H 2000 Thin Solid Films 370 315.

[21] Okamoto S, Kikuchi N, Kitakami O, Miyazaki T, Shimada Y and Fukamichi K 2002 Phys. Rev. B 66024413.

[22] Weisheit M, Schultz L and Fähler S 2004 J. Appl. Phys. 957489.

[23] Klemmer T, Hoydick D, Okumura H, Zhang B and Soffa W A 1995 Scr. Metall. Mater. 33 1793. 